\title{
Seeking Nonspecific Binding: Assessing the Reliability of Tissue Dilutions for Calculating Fraction Unbound
}

\author{
William J. Jusko, Emilie A.G. Molins, and Vivaswath S. Ayyar
}

Department of Pharmaceutical Sciences, School of Pharmacy and Pharmaceutical Sciences, University at Buffalo, Buffalo New York

Received May 14, 2020; accepted July 23, 2020

\begin{abstract}
It has become commonplace $(270+$ article citations to date) to measure the fraction unbound (FrUn) of drugs in tissue homogenates and diluted plasma and then use a Correction Factor Equation (CFE) to extrapolate to the undiluted state. The CFE is based on assumptions of nonspecific binding with experimental use of very low drug concentrations. There are several possible determinants of apparent nonspecific binding as measured by methods such as equilibrium dialysis: true macromolecule binding and lipid partitioning along with receptor, enzyme, and transporter interactions. Theoretical calculations based on nonlinear protein binding indicate that the CFE will be most reliable to obtain FrUn when added drug concentration is small, binding constants are weak, protein concentrations are relatively high, and tissue dilution is minimal. When lipid partitioning is the sole factor determining apparent tissue binding, the CFE should be perfectly accurate. Use of very low drug concentrations, however, makes it more likely that specific binding to receptors and other targets may occur, and thus FrUn may reflect
\end{abstract}

some binding to such components. Inclusion of trapped blood can clearly cause minor to marked discrepancies from purely tissue binding alone, which can be corrected. Furthermore, assessment of the occurrence of ionization/pH shifts, drug instability, and tissue metabolism may be necessary. Caution is needed in the use and interpretation of results from tissue dilution studies and other assessments of nonspecific binding, particularly for very strongly bound drugs with very small FrUn values and in tissues with metabolic enzymes, receptors, and trapped blood.

\section{SIGNIFICANCE STATEMENT}

The use of tissue, plasma, and cell preparations to help obtain fraction unbound and tissue-to-plasma partition coefficients in pharmacokinetics has grown commonplace, especially for brain. This report examines theoretical, physiological, and experimental issues that need consideration before trusting such measurements and calculations.

\section{Introduction}

The "free drug (hormone) hypothesis" (Mendel, 1989; Lin, 2006) is commonly invoked to explain many aspects of drug distribution in pharmacokinetics and pharmacodynamics. Thus, the assessment of unbound drug concentrations in plasma and tissues is often sought. Although such measurements are relatively easy in serum or plasma by ultrafiltration or equilibrium dialysis (except for very highly bound drugs), it is challenging to do this in tissues owing to the need for excision and processing of the samples, which particularly necessitate homogenization. The latter results in a mixture of tissue and blood components and dilution of both the drug and tissue components and, if used for assessing fraction unbound (FrUn), requires extrapolation to the original undiluted values [corrected fraction unbound $\left(c f_{u}\right)$ ]. The employment of diluted tissue homogenates to assess tissue-to-plasma partition coefficients was first proposed by the Hanano laboratory, which used several dilutions for extrapolation to whole tissues (Lin et al.,

This work was supported by National Institutes of Health [Grant R35-131800]. https://doi.org/10.1124/dmd.120.000118.
1982, Harashima et al., 1984). Many tissues and drugs showed good agreement with in vivo values, but some did not.

Kalvass and Maurer (2002) have proposed a Correction Factor Equation (CFE) for adjusting for tissue dilutions in circumstances wherein tissue binding is assumed to be nonspecific, namely linear with drug concentration:

$$
c f_{u}=\frac{\left(\frac{1}{D i l}\right)}{\frac{1}{m f_{u}}-1+\left(\frac{1}{D i l}\right)}
$$

in which measured fraction of drug unbound $\left(m f_{u}\right)$ is the measured FrUn, and $\mathrm{Dil}$ is the single X-fold homogenate dilution factor. This reference has been cited at least 274 times (Web of Science, accessed 08/17/2020), particularly with respect to assessing brain-tissue binding of various CNS drugs and providing implications regarding pharmacology. The methodology is also offered by at least one commercial vendor (Cyprotex, Watertown, MA).

As reviewed (Jusko and Gretch, 1976; Fichtl et al., 1991), numerous tissue components, including albumin, in interstitial fluids can contribute to apparent tissue binding of drugs. Although these will vary with

ABBREVIATIONS: CFE, Correction Factor Equation; $c f_{u}$, corrected fraction unbound; CNS, central nervous system; $C_{T}$, total drug concentration; $C_{w}$, concentration of drug in water; $D_{b}$, concentration of bound drug; DEX, dexamethasone; $D_{f}$, concentration of free drug; Dil, homogenate dilution factor; $D_{t}$, concentration of total drug; $F_{B}$, fraction of plasma/blood in tissue or homogenate; $F_{\text {fat }}$, fraction of tissue or homogenate comprising fat; FrUn, fraction unbound; $f_{u}$, fraction of drug unbound based on a specific measurement or calculation; $f_{u B}$, fraction unbound of drug in whole blood or plasma; $f_{u p}$, unbound fraction in plasma; $f_{u T}$, fraction unbound of drug in whole tissue(s); $F_{w}$, fraction of tissue or homogenate comprising water; $K_{D}$, equilibrium dissociation constant of drug and target (proteins, receptors); $K_{n s}$, nonspecific binding constant of drug; $K_{p}$, lipid/water partition coefficient or in vivo tissue/plasma ratio; MPL, methylprednisolone; PBPK, physiologically based PK PD pharmacodynamic; PK, pharmacokinetics; $P_{t}$, protein concentration; $V_{s s}$, volume of distribution at steady state; $V_{T}$, volume of tissue. 
type of tissue, consider the composition of the brain in particular. The brain consists of about $75 \%$ water, $5 \mathrm{w} / \mathrm{v} \%$ protein, and about $5 \mathrm{w} / \mathrm{v}$ $\%$ lipid. Myelin in normal human brain is largely composed of diverse lipids at 78\%-81\% (by dry weight) (O’Brien and Sampson, 1965). Brain lipids in rats are composed of fractions of total mass of 0.039 neutral lipids, 0.0015 phospholipids, and 0.036 acid phospholipids along with 0.75 water, which are values that differ with species (Rodgers et al., 2012). Furthermore, the brain and other tissues contain numerous transporters, enzymes, and receptors along with blood, which may contribute to apparent tissue binding of drugs as well, particularly at very low drug concentrations (Ballard et al., 1974; Harashima et al., 1984; Fichtl et al., 1991; Fridén et al., 2010; Stieger and Gao, 2015).

The use of the CFE eq. 1 with tissue homogenates and sometimes in diluted plasma (Kalvass et al., 2018) was originally proposed and has been used by many without full assessment of the conditions in which nonspecific binding will occur. The accuracy of the CFE for various experimental conditions and contributions of possible binding and partitioning components of tissues needs further consideration. This report examines the accuracy of the CFE in the context of possible nonlinear protein binding and simple lipid partitioning, considers the potential impacts of trapped blood and drug metabolism, and addresses the possible contributions from specific binding components, such as receptors, enzymes, and transporters.

\section{Theoretical}

Ligand Binding. A standard relationship (Goldstein, 1949) that describes the nonlinear binding of drug to a single type of macromolecule is:

$$
D_{b}=\frac{n \cdot P_{t} \cdot D_{f}}{\left(K_{D}+D_{f}\right)}
$$

in which $D_{b}$ is concentration of bound drug, $D_{f}$ is concentration of free drug, $n$ is the number of binding sites, $P_{t}$ is the protein concentration, and $K_{D}$ is the equilibrium dissociation constant of drug and target (proteins, receptors). The concentration of total drug $\left(D_{t}\right)$ is:

$$
D_{t}=D_{b}+D_{f}
$$

The fraction of drug unbound based on a specific measurement or calculation $\left(f_{u}\right)$ is typically measured experimentally as $D_{f} / D_{t}$ and follows from eqs. 2 and 3 as:

$$
f_{u}=\frac{\left(K_{D}+D_{f}\right)}{\left(K_{D}+n \cdot P_{t}+D_{f}\right)}
$$

For calculation purposes, it is necessary to generate values of $f_{u}$ from known concentration $D_{t}$ as the positive root of the quadratic:

$$
f_{u}=\frac{-b+\sqrt{b^{2}-4 a c}}{2 a}
$$

in which: $a=D_{t}, b=\left[K_{D}+n \cdot P_{t}-D_{t}\right]$, and $c=-K_{D}$.

Equation 4 includes the four indicated determinants of actual protein binding over the full range of possible drug and protein concentrations for one type of binding macromolecule $\left(P_{t}\right)$. The common definition of nonspecific binding is the condition in which $D_{f}$ in eq. 4 is very small and thus negligible in relation to both $K_{D}$ and $n \cdot P_{t}$, yielding:

$$
f_{u}=\frac{K_{D}}{\left(K_{D}+n \cdot P_{t}\right)}
$$

This equation leads to the CFE relationship (eq. 1) in which Dil accounts for differences in $n \cdot P_{t}$ for the diluted homogenate (Kalvass and Maurer, 2002).
Lipid Partitioning. The model shown in Fig. 1 depicts an equilibrium dialysis cell in which the tissue homogenate consists of lipid (fat) suspended in an aqueous liquid on one side of a semipermeable membrane with an aqueous medium on the other side. The lipid/aqueous partition coefficient is:

$$
K_{p}=\frac{C_{f a t}}{C_{w}}
$$

The measured total drug concentration $\left(C_{T}\right)$ in the homogenate side at equilibrium is:

$$
C_{T}=C_{w} \cdot F_{w}+C_{f a t} \cdot F_{f a t}
$$

in which $F_{w}$ is the fraction of tissue or homogenate comprising water, $F_{f a t}$ is the fraction of tissue or homogenate comprising fat, and $F_{w}=1-$ $F_{\text {fat }}$. This leads to:

$$
C_{T}=C_{w} \cdot\left[1+F_{f a t}\left(K_{p}-1\right)\right]
$$

Since in this situation, $f_{u}=C_{w} / C_{T}$, then

$$
f_{u}=\frac{1}{\left[1+F_{f a t}\left(K_{p}-1\right)\right]}
$$

With dilution ( $D i l)$ of the homogenate changing values of $F_{\text {fat }}$, the relationship between the measured homogenate $\left(m f_{u}\right)$ and corrected fraction unbound $\left(c f_{u}\right)$ in whole tissue is:

$$
c f_{u}=m f_{u} \cdot\left[\frac{1+\left(\frac{F_{\text {fat }}}{D i l}\right)\left(K_{p}-1\right)}{1+F_{\text {fat }}\left(K_{p}-1\right)}\right]
$$

This equation is functionally equivalent to CFE eq. 1 .

Correction for Trapped Blood. The inclusion of trapped blood or plasma in a tissue homogenate is unavoidable except by carrying out either whole-body or organ perfusion prior to or just after sacrifice of an animal. This problem is well recognized in analyzing tissue samples in physiologically based pharmacokinetics (PBPK) studies (Khor and Mayersohn, 1991). To correct tissue homogenate-binding measurements for this problem, it is necessary to first obtain FrUn in undiluted plasma or blood [fraction unbound of drug in whole blood or plasma $\left.\left(f_{u B}\right)\right]$. In turn, the expected FrUn attributed to true tissue in the corrected tissue homogenate $\left(c f_{u}\right)$ can be calculated.

Consider the diagram in Fig. 2 to represent the composition of the whole tissue [volume of tissue $\left(V_{T}\right)$ ], with $F_{B}$ as the fraction of plasma/ blood in tissue or homogenate and $F_{T}$ as the fraction of actual tissue separate from blood. The following mass balance would hold:

$$
\text { Amount in Tissue } \begin{aligned}
\left(A_{T}\right)= & \left(C_{f}+C_{b B}\right) \cdot F_{B} \cdot V_{T} \\
& +\left(C_{f}+C_{b T}\right) \cdot F_{T} \cdot V_{T}
\end{aligned}
$$

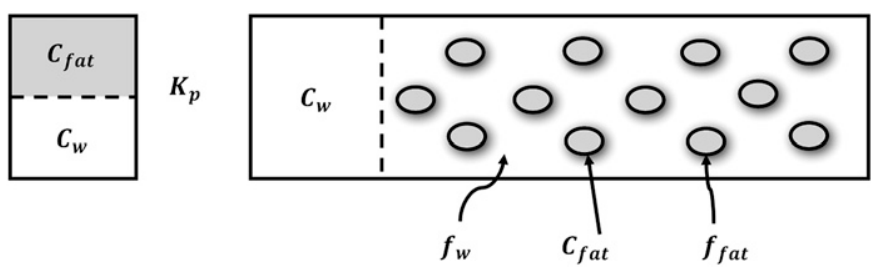

Fig. 1. Lipid partitioning: model of determinants of tissue drug distribution and apparent binding for simple lipid [concentration of drug in fat $\left(C_{f a t}\right)$ to aqueous $\left(C_{w}\right)$ partitioning. The partition coefficient $K_{p}=C_{f a t} / C_{w}$. For equilibrium dialysis, the total tissue homogenate concentration $C_{T}=C_{w} \cdot F_{w}+C_{f a t} \cdot F_{f a t}$, in which $F_{w}$ and $F_{f a t}$ are the fractional aqueous and fat contents. 


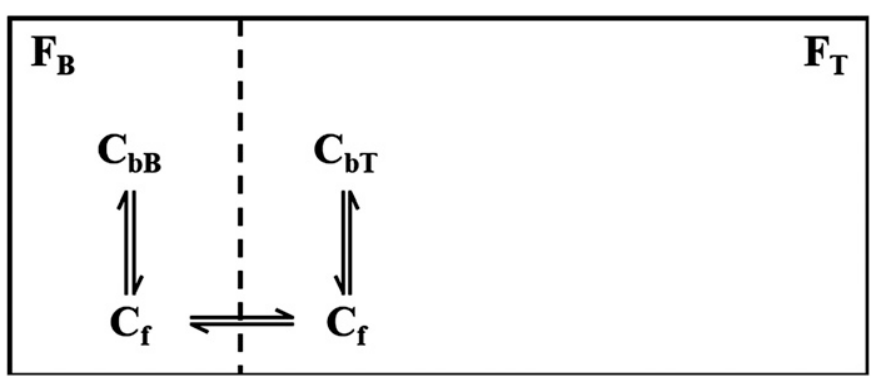

Fig. 2. Model for relationship of trapped blood in tissues and role of binding. Concentration of free drug $\left(C_{f}\right)$ is assumed to equilibrate with concentration of bound drug in blood $\left(C_{b B}\right)$ and concentration of bound drug in tissue $\left(C_{b T}\right) . F_{B}$ and fraction of actual tissue separate from blood $\left(F_{T}\right)$ are noted. $V_{T}$, volume of tissue.

in which $C_{f}$ is concentration of free drug, $C_{b B}$ is concentration of bound drug in blood, and $C_{b T}$ is concentration of bound drug in tissue. The total tissue-homogenate concentration $\left(C_{T}\right)$ is:

$$
C_{T}=\frac{A_{T}}{V_{T}}=\left(C_{f}+C_{b B}\right) \cdot F_{B}+\left(C_{f}+C_{b T}\right) \cdot F_{T}
$$

The $c f_{u}$ is:

$$
c f u=\frac{C_{f}}{C_{T}}=\frac{C_{f}}{\left(C_{f}+C_{b B}\right) \cdot F_{B}+\left(C_{f}+C_{b T}\right) \cdot F_{T}}
$$

Division by $C_{f}$ and recognizing the FrUn relationships for blood and remaining tissue:

$$
f_{u B}=\frac{C_{f}}{C_{f}+C_{b B}}
$$

and

$$
f_{u T}=\frac{C_{f}}{C_{f}+C_{b T}}
$$

yields:

$$
c f u=\frac{1}{\frac{F_{B}}{f_{u B}}+\frac{F_{T}}{f_{u T}}}
$$

Rearrangement results in:

$$
f_{u T}=\frac{F_{T}}{\frac{1}{c_{f u}}-\frac{F_{B}}{f_{u B}}}
$$

Note that these calculations need consistency in using only either plasma binding and fraction of plasma in tissues or corresponding whole-blood binding and fractions in these equations. They assume that there will be no cross-interference for binding between plasma/blood and tissue components in the homogenate. Because of the negative sign in eq. 17 , this equation will only operate properly when $c f_{u}$ falls between $f_{u B}$ and $f_{u T}$. Note that eq. 17 will apply after the CFE correction of the tissue homogenate is made.

The composition of the vascular space in brain has been assessed using several methods as reviewed (Friden et al., 2010). Residual blood in rat brain comprises water 10.3 , plasma proteins 7.99 , and erythrocytes $2.13 \mu \mathrm{l} / \mathrm{g}$ brain, namely about $2 \%$ of brain mass. They introduced a correction model for calculating the brain/plasma-unbound partition coefficient.
Receptor Binding. Drug bound in presence of enzymes, receptors, transporters, or other targets with strong binding in tissues occurring along with nonspecific binding can be described as:

$$
D_{b}=\frac{B_{\max } \cdot D_{f}}{\left(K_{D}+D_{f}\right)}+K_{n s} \cdot D_{f}
$$

in which $B_{\max }$ is total receptor concentration, $K_{D}$ is the drug-receptor dissociation constant, and $K_{n s}$ is the nonspecific binding constant of drug (Hazra et al., 2007).

\section{Methods}

Previous experimental data for dexamethasone (DEX) binding to receptors in rat liver cytosol were obtained (Hazra et al., 2007) using a developed radioligand-binding assay (Boudinot et al., 1986) with some modifications. Binding assessments of methylprednisolone (MPL) and DEX in rat tissue homogenates were recently carried out (Ayyar et al., 2019b).

\section{Results}

Ligand Binding. Simulations were performed using eqs. 4 and 5 to assess the expected values of FrUn in relation to varying drug and protein concentrations for hypothetical drugs with weak $K_{D}=10^{-4} \mathrm{M}$, moderate $K_{D}=10^{-5} \mathrm{M}$, and strong $K_{D}=10^{-7} \mathrm{M}$ equilibrium dissociation constant values. The standard practice in applying the CFE for brain tissue is to employ an added drug concentration of 1 or $5 \mu \mathrm{M}$ (Kalvass et al., 2007; Friden et al., 2011). To mimic the presence of a very low or trace drug concentration (eq. 6) and apparent nonspecific binding, a drug concentration of $0.01 \mu \mathrm{M}$ was compared with an assumed added concentration of $10 \mu \mathrm{M}$. Ultimately, the ability of the CFE (eq. 1) to recover the "true" FrUn was assessed. For these and subsequent simulations, we assumed one class of binding proteins as described by eq. 2 , with the protein having the molecular weight of albumin $(69,000)$

Figure 3 shows values of FrUn in relation to a wide range of protein concentrations for drugs with three values of $K_{D}$ in which the tracer and experimental drug concentrations were used in operation of eqs. 4 and 5 . As expected, the lowest $K_{D}$ value produced strongest binding and the lowest values of FrUn, ranging from about 0.5 at very low-protein concentrations to $f_{u}=0.0001$ at the $P_{t}$ of $10^{-3} \mathrm{M}$. The latter is approximately the total protein concentration in brain of $6.9 \%$. The FrUn is similar for the two drug concentrations at the higher protein concentrations and more weakly bound drugs. However, there is increasing divergence of FrUn values for the two drug concentrations when protein concentrations are low, especially for the more strongly bound compound. It can be noted that when starting with a set $D_{t}$ concentration, there will be generation of lower FrUn values because of reductions in $D_{f}$ as binding increases.

Figure 4 provides simulations in which FrUn was calculated for a wide range of total drug concentrations but when values were determined for relatively low $10^{-6} \mathrm{M}$ and high $10^{-4} \mathrm{M}$ protein concentrations. The FrUn values are of course lowest when $K_{D}$ was strongest $\left(10^{-7} \mathrm{M}\right)$ and protein concentrations were higher. The values of FrUn were nonlinear with drug concentration as expected, but all of the profiles show constant FrUn values for a wide range of very low drug concentrations. The determination of possible consistency of FrUn thus requires consideration of all three contributing factors of $D_{t}, P_{t}$, and $K_{D}$.

Figure 5 depicts the differences expected in FrUn over a range of protein concentrations when added drug concentrations are 10 versus $0.01 \mu \mathrm{M}$. The latter concentration will produce least saturation of binding and best reflect strongest (lowest FrUn) binding conditions. 
Protein concentration (g/dL)

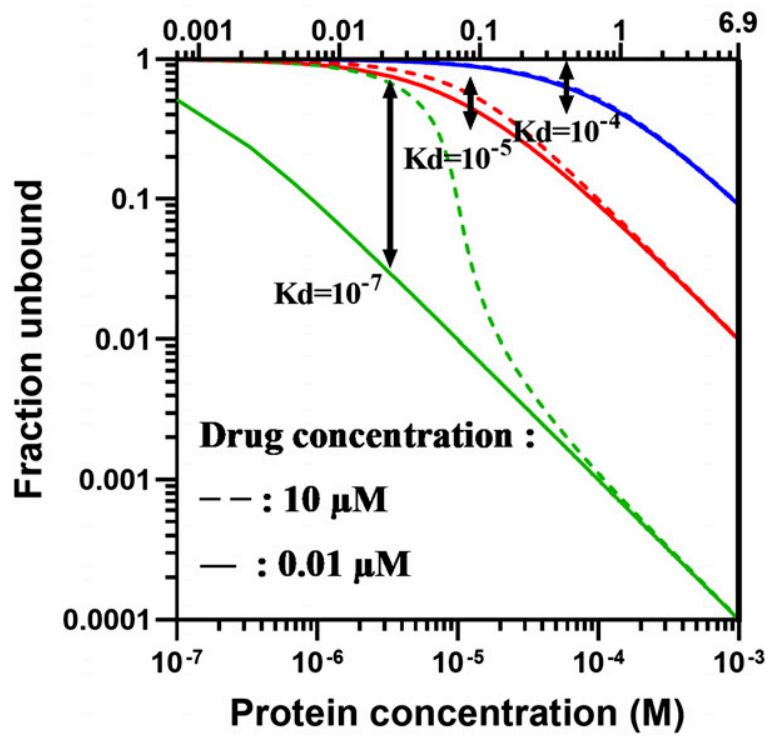

Fig. 3. Ligand binding: relationship of $f_{u}$ vs. $P_{t}$ for compounds with three different $K_{D}$ values according to eq. 2 for the indicated values of $D_{f}$.

The smallest differences are found at relatively high protein concentrations $\left(P_{t}=10^{-4}\right.$ to $\left.10^{-3} \mathrm{M}\right)$, and substantial differences are found when protein concentrations are very low. Interestingly, the drug with moderate binding $\left(K_{D}=10^{-5} \mathrm{M}\right)$ exhibited a wider range of FrUn ratios closest to a $1 \%$ difference compared with the others.

Figure 6 demonstrates the ability of the CFE to recover the true FrUn for the three binding constants over the range of protein concentrations when it was assumed that the drug concentration added was $10 \mu \mathrm{M}$, and the tissue was diluted 3-fold as is commonly done. In this case, the true FrUn was calculated using eqs. 4 and 5 for a given drug and protein concentration, and a "measured" FrUn was obtained with the $P_{t}$ reduced to one-third. In turn, the calculated or experimental FrUn was generated using eq. 1 . The graph provides the ratio of the $c f_{u} /$ true $f_{u}$. The CFE was found to function well for drugs with all $K_{D}$ values when protein concentrations were high but diverged appreciably for the stronger binding compound as protein concentrations decreased.

These simulations indicate that for simple binding alone, the reliability of the CFE will depend strongly on utilizing the lowest possible drug concentration to seek linearity and greatest degree of binding but will be most dependable for more weakly binding drugs and when the binding protein is present in relatively higher concentrations. These conditions are helped by diluting the tissue homogenate as little as possible.

Lipid Partitioning. Simulations of apparent FrUn were performed based on the assumption that equilibrium dialysis would be performed (Fig. 1) using diluted tissue homogenates with a classic partition coefficient [lipid/water partition coefficient or in vivo tissue/plasma ratio $\left(K_{p}\right)$ ] and lipid partitioning solely responsible for drug concentrations in the aqueous $\left(C_{w}\right)$ and homogenate $\left(C_{T}\right)$ chambers. Figure 7 shows the relationship of apparent FrUn versus the fraction of fat in the homogenate for differing values of $K_{p}$. Although the $F_{f a t}$ axis is scaled to 1.0 , practical values for undiluted tissue would range from about 0.05 for brain to 0.8 for adipose tissue (Rodgers et al., 2012). As expected, higher values of $K_{p}$ produce smaller values of FrUn. It can be noted that the largest $K_{p}$ represents a $\log \mathrm{P}$ of 6 , and the range includes a wide array of drugs. These simulations assume that the drug is not ionized where the situation becomes more complicated.

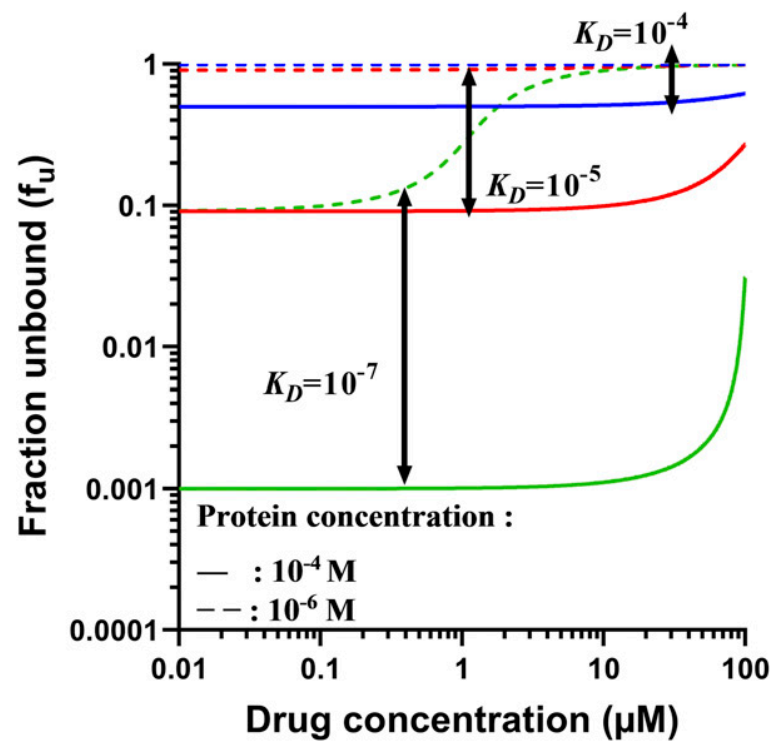

Fig. 4. Ligand binding: relationship of $f_{u}$ vs. $D_{f}$ for two $P_{t}$ values according to eq. 2 .

Figure 8 was constructed in a similar manner to Fig. 6 in providing an assessment of how well the CFE would recover the true FU in a situation in which $10-\mu \mathrm{M}$ concentrations of drug were added to a tissue homogenate diluted 3-fold. A "measured" FrUn was generated using eq. 10 for the dilution, and then eq. 1 was employed to obtain the calculated $c f_{u}$. The ratios of $c f_{u}$ to true $f_{u}$ are graphed in relation to $F_{\text {fat }}$ in Fig. 8. For all values of $K_{p}$ and $F_{f a t}$, the CFE produced exact predictions of the true FrUn. Since lipid partitioning was assumed to be a linear process with constant $K_{p}$ values, this would be true for any added drug concentration.

Presence of Trapped Blood/Plasma. Figure 9 shows simulations using eq. 17 of possible effects of trapped blood/plasma in tissue homogenates used to assess tissue binding. The calculations assume that the true $f_{u T}=0.05$ and consider the effects of various degrees of

\section{Protein concentration (g/dL)}

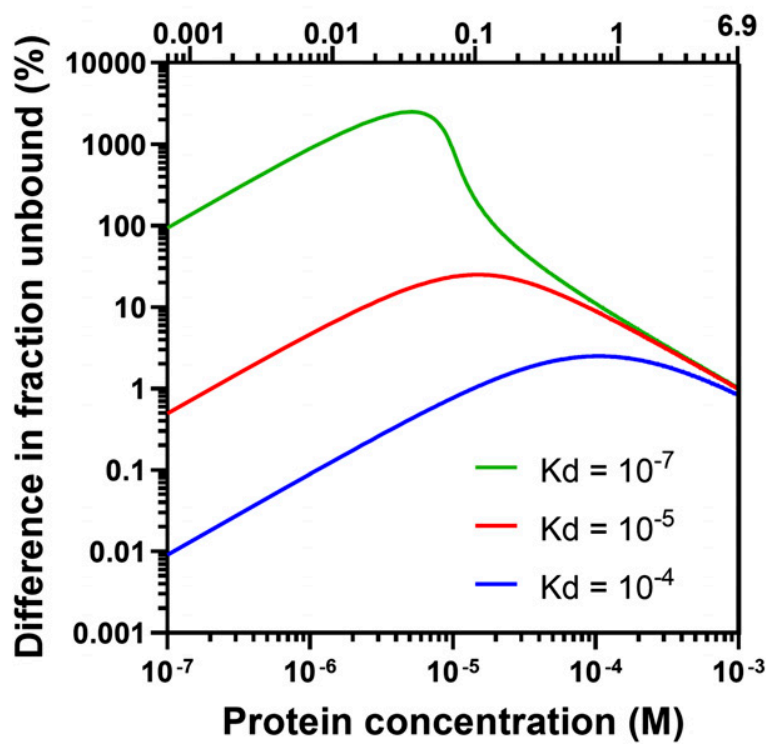

Fig. 5. Ligand binding: differences in $f_{u}$ in relation to $P_{t}$ for compounds with three $K_{D}$ values when drug concentrations are 10 and $0.01 \mu \mathrm{M}$ according to eq. 2 . 


\section{Protein concentration $(\mathrm{g} / \mathrm{dL})$}

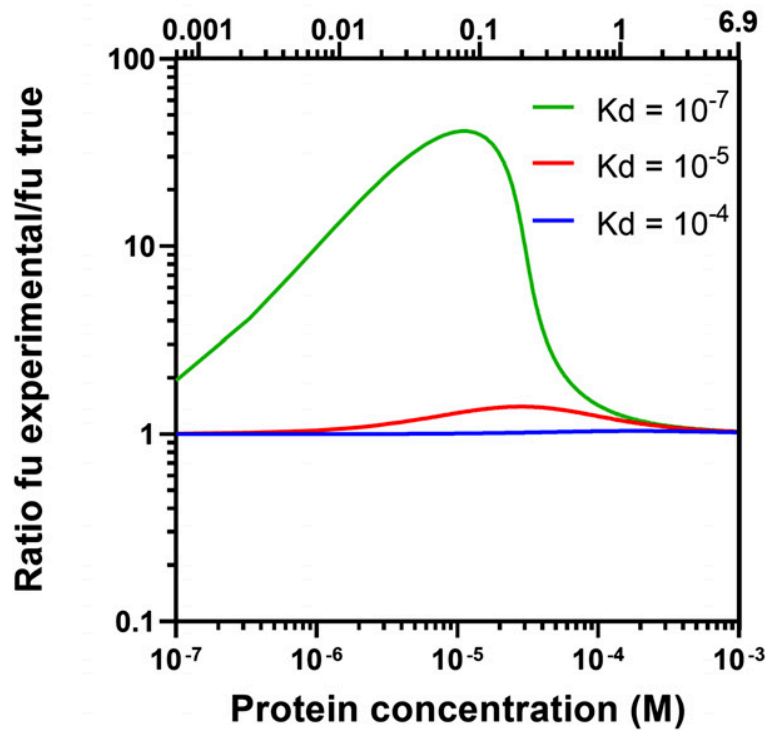

Fig. 6. Ligand binding: ratio of experimental $f_{u}$ to true $f_{u}$ in relation to $P_{t}$ for compounds with three $K_{D}$ values. The experimental $f_{u}$ was calculated based on the CFE (eq. 1) assuming a Dil of 3.

blood/plasma-binding $f_{u B}$ values as denoted for the fractions of $F_{B}$ ranging from 0 to 0.5 as shown in the graph. It can be seen that deviations from true $f_{u T}$ values can vary in both the positive and negative directions depending on $f_{u B}$ in relation to $f_{u T}$. When blood/plasma binding is less than tissue binding, there is a dilution effect from the trapped blood that skews the deviation in the positive direction. When plasma binding is stronger than tissue binding, the deviation is skewed in the negative direction, sometimes considerably when blood FrUn is very strong. When they are similar, there is no correction needed, as indicated by the horizontal line in the graph. This simulation shows only one set of possibilities since the variable degrees of binding and tissue compositions will all contribute to a wide range of possible deviations from true values. It can be noted that some drugs have an unbound fraction in plasma $\left(f_{u p}\right)$ as low as 0.000012 (venetoclax), which requires special approaches for measurement (Kalvass et al., 2018).

Specific Target Binding. An example of the combination of specific receptor binding and nonspecific binding that can occur for many ligands and tissues is shown in Fig. 10. This graph depicts an experimental assessment of dexamethasone binding to components of rat liver cytosol (Hazra et al., 2007). To quantitate joint glucocorticoid receptor binding and nonspecific binding, the radiolabeled drug $\left(\mathrm{H}^{3}\right)$ is added along with a range of $D_{t}$ values, bound drug-receptor complex $\left(D_{b}\right)$ is separated by ultracentrifugation, and the relationship between $D_{b}$ and $D_{f}$ is assessed. The contribution of $K_{n s}$ is determined by saturating receptor binding with added unlabeled drug in very high concentrations ( $100 \times$ the values on the $y$-axis) in companion samples. The operative binding equation in this case is eq. 18 .

Joint fitting of the data when there is a range of low and very high drug concentrations allows identification of $B_{\max }, K_{D}$, and $K_{n s}$. This example demonstrates that the common practice of screening tissue homogenates for calculation of FrUn using a single very low drug concentration does not necessarily reflect nonspecific binding but may include measurement of binding to possible receptors or other biologic targets.

Presence of Metabolic Enzymes or Drug Instability. Figure 11 depicts the metabolic and binding assessments of the corticosteroid MPL in diluted homogenates freshly prepared from male rat livers.

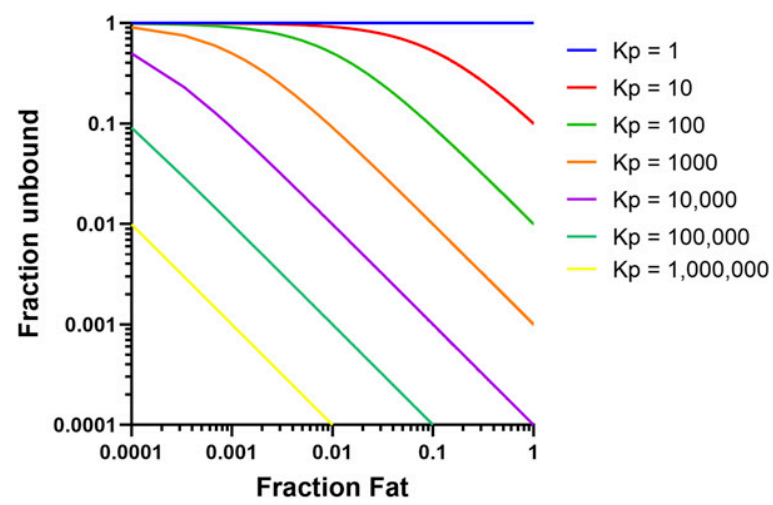

Fig. 7. Lipid partitioning: relationship between $f_{u}$ and $F_{f a t}$ for compounds with the listed $K_{p}$.

The metabolic loss rate of MPL was more rapid in the less diluted homogenates but linear with time and dilution. These data were used to assess hepatic clearance of MPL (Ayyar et al., 2019b). To quantitate the FrUn, the tissue homogenates were diluted (3- to 10-fold), and drug was added at a concentration of $10 \mu \mathrm{g} / \mathrm{ml}$. Preliminary validation experiments performed at $1 \mu \mathrm{g} / \mathrm{ml}$ yielded a similar $D_{b} / D_{f}$ value as compared with $10 \mu \mathrm{g} / \mathrm{ml}$, suggesting concentration-independent binding within this range. These concentrations correspond well with observed MPL liver exposures in our in vivo PK-pharmacodynamic assessments in rats (Ayyar et al., 2019a). Then samples were loaded into ultrafiltration devices and incubated at $37^{\circ} \mathrm{C}$ for a predetermined time period (to achieve an equilibrium in binding), free and bound drug was separated by ultrafiltration at $37^{\circ} \mathrm{C}$, and the relationship between values of the binding ratio $D_{b} / D_{f}$ and the $D i l$ were assessed at each dilution. The observed linear relationship can be derived from eq. 2 when $D_{f}$ is very low:

$$
\frac{D_{b}}{D_{f}}=\frac{n \cdot P_{t}}{K_{D}} \cdot \frac{1}{D i l}
$$

in which the slope $=n \cdot P_{t} / K_{D}$.

With back-extrapolation to unity (i.e., an undiluted tissue state), $f_{u T}$ was computed using the relationship (Lin et al., 1982; Ayyar et al., 2019b):

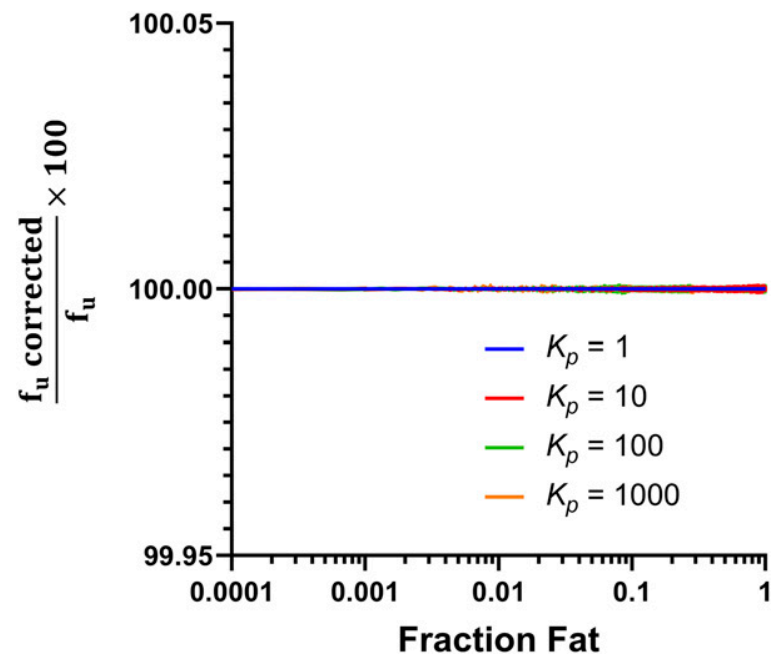

Fig. 8. Lipid partitioning: relationship of $c f_{u}$ using the dilution equation (eq. 1) to the true $f_{u}$ (eq. 11) vs. $F_{\text {fat }}$ for compounds with the listed $K_{p}$ using a drug concentration of $10 \mu \mathrm{M}$. 


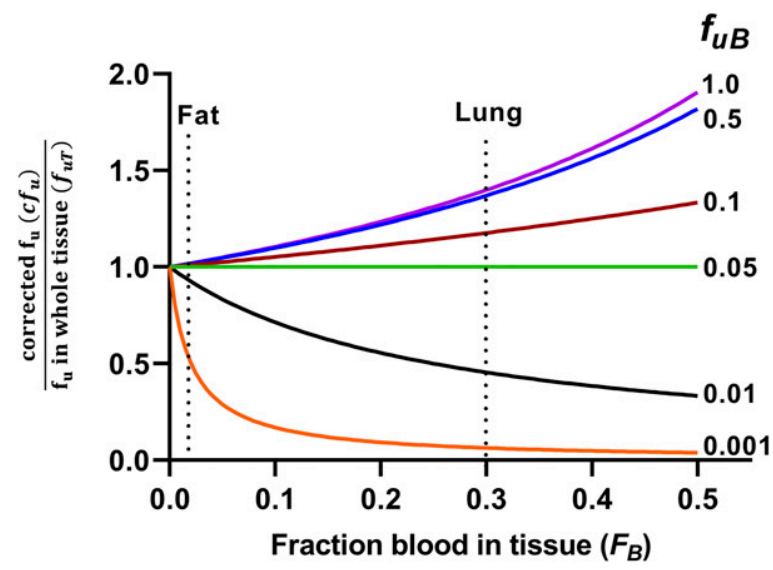

Fig. 9. Deviations from true tissue-binding $f_{u T}$ with varying $F_{B}$ and various degrees of blood/plasma binding (as indicated by $f_{u B}$ values) present in the tissue homogenate.

$$
f_{u T}=\frac{1}{\frac{D_{b}}{D_{f}}+1}
$$

As shown in Fig. 11, MPL binding was proportional to the total protein concentration in liver homogenates across 3- to 10-fold dilutions. Employing the method in eq. 2, the $f_{u T}$ for MPL in liver was $0.162 \pm$ 0.01 . Since most screening studies employ a single dilution, the measured FrUn was also corrected to the undiluted state using eq. 1. Using only the 3 -fold dilution values, the CFE method yielded a value of $0.150 \pm 0.01$. The use of several dilutions of homogenates is advantageous in providing more robust data for extrapolation and confirming linearity of binding with varying tissue dilutions. The plasma binding of MPL at $37^{\circ} \mathrm{C}$ was linear with moderate binding of $61 \%\left(f_{u p}=\right.$ 0.39 ). Our studies of corticosteroids are not complicated by changes or differences in tissue $\mathrm{pH}$ since these are neutral compounds. It can be noted that the published calculation methods (Poulin and Theil, 2002; Berezhkovskiy, 2004; Rodgers and Rowland, 2006) for estimating tissue:plasma ratios yielded reasonable estimates of $K_{p}$ in muscle and lung as compared with our directly measured values, but estimates in liver differed appreciably (Ayyar et al., 2019b). Although we did not, at the time, recognize the importance of trapped blood, recalculation using the CFE and eqs. 16 and 17 (blood/plasma partition coefficient equal to 1) revealed that tissue FrUn values for MPL are essentially the same in muscle but change from 0.17 to 0.14 in lung, 0.16 to 0.13 in male liver, and 0.063 to 0.055 in female rat liver. Corresponding values for DEX also changed from 0.12 to 0.11 in lung and 0.066 to 0.059 in liver (Ayyar et al., 2019b).

\section{Discussion}

Binding to plasma and tissues is a major determinant of drug distribution in the body and thus plays an important role in pharmacokinetics. The ratio of $f_{u p}$ and $f_{u T}$ generally defines the $K_{p}$, an important parameter in PBPK that determines total drug concentrations within tissues and the volume of distribution at steady-state $\left(V_{s s}\right)$. Since $K_{p}$ is often governed by reversible binding to macromolecules in blood and tissues, in vitro assessments of drug binding in these media are used to estimate tissue $K_{p}$ values in vivo. However, in vivo drug uptake into tissues may be more complicated because the "permissive" availability from both plasma-binding proteins and transporter-mediated tissue influx can occur (Herve et al., 1994). Transporter-mediated efflux is also commonplace, particularly in brain and liver.

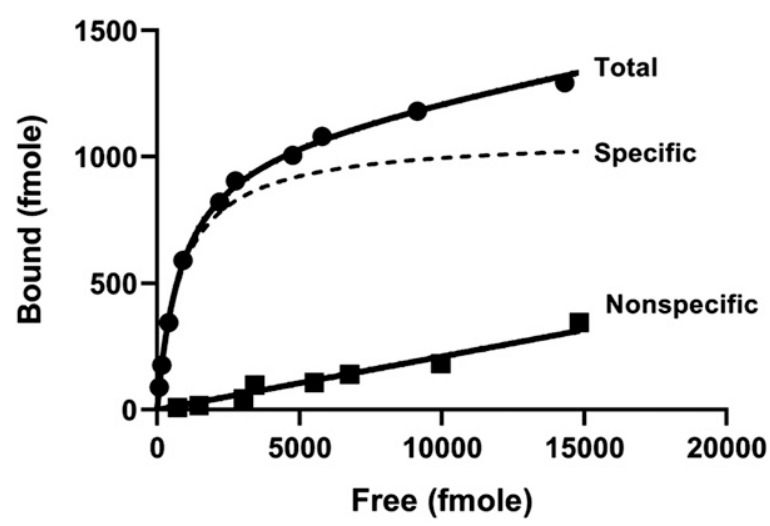

Fig. 10. Specific target binding. Relationship of $D_{b}$ vs. $D_{f}$ in an experiment where $\mathrm{H}^{3}$-dexamethasone binding to glucocorticoid receptors in rat liver cytosol was assessed for calculation of $B_{\max }$ and affinity $\left(K_{D}\right)$. The free-drug concentration scale for nonspecific binding was about 100-fold higher than that labeled. Joint assessment of total and $K_{n s}$ was necessary to resolve all binding parameters. In this study, values of $B_{\max }=1079 \mathrm{fmol}, K_{D}=844 \mathrm{fmol}$, and $K_{n s}=0.021 \mathrm{fmol}$ were calculated. From Hazra et al. (2007).

Numerous tissue components can bind drugs, including immunophilins, phospholipids, microsomes, mitochondria, DNA, and others (Jusko and Gretch, 1976, Fichtl et al., 1991). Organs like muscle and heart contain contractile proteins that bind cardiac glycosides. A PBPK model
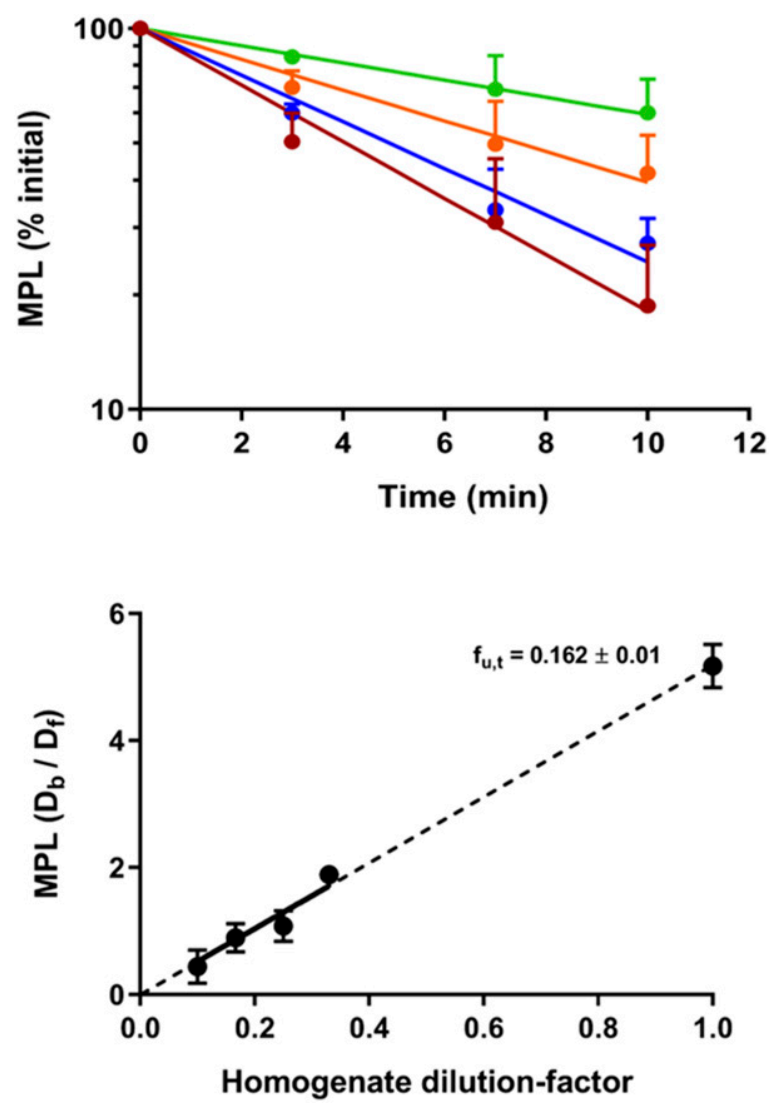

Fig. 11. (Top) Time course of in vitro stability of MPL in liver homogenates prepared at 3- (red), 4- (blue), 6- (orange), and 10-fold (green) dilutions from freshly harvested male livers. Symbols are the mean \pm S.D. $(n=3$ per time point $)$. (Bottom) Binding of MPL in homogenates prepared from male rat liver at initial concentrations of $10 \mu \mathrm{g} / \mathrm{ml}$. Symbols depict the mean \pm S.D. of the binding ratios $\left(D_{b} / D_{f}\right)$ across four dilutions of tissue homogenate. The dashed line represents the best-fit line (eq. 19) extrapolated to an undiluted state of tissue $($ Dil $=1)$. From Ayyar et al. (2019b). 
for doxorubicin included nonlinear binding to DNA and cardiolipin (Gustafson et al., 2002). Many compounds exhibit nonlinear targetmediated binding and disposition (Mager and Jusko, 2001), including vildagliptin that binds to dipeptidyl peptidase IV both in plasma and tissues (Landersdorfer et al., 2012). Albumin and globulins are both found in interstitial fluids at concentrations that are usually about onehalf of that in plasma (Jusko and Gretch, 1976). Interestingly, the methods of estimating tissue $K_{p}$ values attribute involvement of the three types of tissue lipids and thus invoke simple partitioning (Rodgers and Rowland, 2006). Uncertainties exist if the tissue dilution and low drug concentrations used in measurements are adequate to allow for extrapolations of FrUn to whole-tissue values. Notably, our simulations - on one hand - argue for use of low drug concentrations and minimal dilutions (higher protein concentrations); our demonstration-on the other hand-of receptor binding (Fig. 10) indicates that drug concentrations considerably higher than $K_{D}$ values (usually nanomolars) are needed to see linearity.

Our simulations that assess the CFE method for drug binding to tissue components with possibly nonlinear binding demonstrate generally good but not perfect agreement of the methodology with theory (Figs. 3-6). Similar simulations assessing the role of protein concentration affecting either FrUn or fraction bound were published (Jusko and Gretch, 1976; Wan et al., 2007). All show that there is a range of protein concentrations (and thus dilutions) that will produce similar FrUn values, but caution is needed that such range may not be foreseen without experimentation. This was done in some studies in tissues (Lin et al., 1982; Wan et al., 2007; Ayyar et al., 2019b) and plasma (Kalvass et al., 2018).

If fat distribution with linear partition coefficients accounts for tissue "binding," then the CFE method works perfectly in theory (Fig. 8). Body tissues of rats contain various percentages of neutral lipids, with the fat content ranging from about $4 \%$ in brain to $86 \%$ in adipose tissues (Rodgers et al., 2012). The contribution of the $F_{f a t}$ to values of FrUn in tissues varies with the partition coefficient and fat content (Fig. 7). Drugs exhibit a wide range of $\log \mathrm{P}$ and $\log \mathrm{D}$ values, and this factor is considered in computational methods that predict tissue partition coefficients. Our simulations extend to tissue distribution expectations for compounds with very high $\log \mathrm{P}$ values (6) in which the in vivo $K_{p}$ is determined by the relative concentrations of lipid in plasma/blood versus tissue. This appears to produce an upper limit in in vivo $K_{p}$ values (Haddad et al., 2000). However, the CFE appears to work reasonably well in brain for many neutral compounds owing to its low-protein, high-lipid, and minimal-trapped blood contents.

Trapped blood offers a major possible artifact when examining tissue samples from in vivo studies to obtain $K_{p}$ values (Khor and Mayersohn, 1991). It is commonplace in PBPK studies to correct for trapped blood using values for fractional tissue blood space (Bernareggi and Rowland, 1991). This is clearly a possible artifact for using tissue homogenates and slices to assess binding as well. The presence of albumin (and perhaps other plasma proteins) at a concentration of about $0.8-1.0 \mu \mathrm{M}$ in brain homogenates is considered to reflect contamination from trapped blood (Longhi et al., 2011; Loryan et al., 2016). Mouse-brain homogenates were found to contain about $1 \%$ trapped blood using chromium-labeled red blood cells (Garg and Balthasar, 2009). Except for one report (Friden et al., 2010), this problem has largely gone unrecognized for tissue homogenates. Although this artifact seemingly can be obviated by perfusion of the animal organ or whole body with saline (PBS) before processing the tissue (Taves et al., 2010), this process produces a dilution factor if the perfusion fluid remains "trapped" in the tissue (similar to 0 FrUn binding shown in Fig. 9). Studies with 36 compounds were carried out in rats in which whole-body perfusions with PBS were carried out before collection of 14 sets of tissues (Berry et al., 2010).
Diluted tissue homogenates were analyzed, and the CFE was applied to obtain $f_{u T}$ and ultracentrifugation to measure $f_{u p}$. Values of $V_{s s}$ factoring in the blood/plasma partition coefficient $(B / P)$ ratio were generated by:

$$
V_{s s}=V_{\text {plasma }}+\sum \frac{f_{u p} /(B / P)}{f_{u t}}
$$

These summed Vss values were within 2-fold of PK-calculated values for $77 \%$ of neutral compounds and for $61 \%$ for all compounds (neutral, acids, bases, zwitterions). There is clearly room for improvement in using tissue homogenates for predicting tissue binding and $V_{s s}$ values.

Our assessments pertain to simple factors that control tissue binding and accuracy of the CFE method and do not account for additional determinants, such as ionization for acids and bases, relevant $\mathrm{pH}$ gradients, subcompartments, and other differences among tissues. Ionization can be accounted for when using computational methods when the pKa of the drug is employed (Poulin and Theil, 2000; Berezhkovskiy 2004; Rodgers and Rowland, 2006; Poulin, 2015). Brain-slice measurements of tissue partition coefficients that preserve tissue architecture are considered more reliable than tissue homogenates owing to the better integrity of drug-pH partitioning (Friden et al., 2007, 2011). These authors also provide useful insights into tissue subcompartments that may attain far differing drug concentrations than those indicated by in vitro measurements that destroy tissue integrity. Weak acids will be primarily found in interstitial fluids owing to their extensive ionization that reduces cellular access. Weak bases that are more highly ionized at lower $\mathrm{pH}$ values will concentrate in lysosomes with low $\mathrm{pH}$ (4.5-5.5) compared with the cytosol $\mathrm{pH}$ of 7.0-7.5 (Yokogawa et al., 2002; Trapp et al., 2008).

The role of trapped blood in skewing use of homogenates for tissue binding will, of course, vary with the type of tissue or organ and its content of trapped blood (Fig. 9). Although the brain and muscle contain the smallest fractions of trapped blood, the compounds that are most effective in the CNS tend to be lipophilic weak bases with high degrees of plasma protein and lipid binding. Large deviations from the true tissue FrUn are expected even with small percentages of trapped blood when plasma or blood FrUn is much larger than tissue FrUn (Fig. 9). Most of the 42 CNS-active compounds assessed in mouse plasma and brain homogenates generally showed $f_{u T} \geq f_{u p}$ values (Wan et al., 2007). Furthermore, 108 diverse compounds demonstrated a strong correlation $(\mathrm{r}=-0.78)$ of $f_{u T}$ with cLogP values.

Although the CFE method is most commonly applied to brain homogenates, the brain has ATP-binding cassette transporters (Stieger and Gao, 2015; Gomez-Zepeda et al., 2019). Several studies have compared $K_{p}$ values obtained from brain homogenates with in vivo measured values using a variety of compounds. Good concordance for nine compounds was found when passive CNS distribution was expected, but appreciable overestimates of $f_{u \text {-plasma }}$ to $f_{u \text {-brain }}$ ratios occurred for compounds with an active efflux mechanism (Kalvass and Maurer, 2002). Brain homogenate FrUn values were assessed for 56 compounds, of which 13 were neutral compounds not subject to $\mathrm{pH}$ considerations (Friden et al., 2011). The ratios of observed to predicted FrUn values were within $20 \%$ for eight neutral compounds and off by varying degrees for the five others. Assessments of brain distribution of CNS drugs for 33 compounds showed that 23 exhibited unbound brainto-plasma ratios that were within 3 -fold (Kalvass et al., 2007). Most were weak bases, but the acidic and neutral compounds showed closer agreement. Thus, there is considerable uncertainty in use of tissuebinding measurements to estimate in vivo $K_{p}$ values.

Drug loss in tissue homogenates prepared from metabolizing organs can significantly impact measurements of FrUn in tissue when times of processing and equilibrium dialysis are factors. For example, brain tissue 
in human and rat can convert alprazolam to active hydroxy metabolites (Agarwal et al., 2008). This effect was apparent during our assessments of MPL binding, particularly with use of liver homogenates (Fig. 11). To reduce the impact of drug metabolism or degradation in various media, some homogenate-binding studies are conducted at $4^{\circ} \mathrm{C}$ (Lin et al., 1982; Berry et al., 2010). However, the unbound fractions for several drugs tend to increase at lower temperatures (Ballard, 1974). In addition to metabolic instability, chemical instability may need confirmation. Sirolimus is unstable, with a half-life of about 15 hours in rat whole blood, which likely will also occur in tissues and homogenates (Ferron and Jusko, 1998).

Methods that are frequently used to assess in vitro drug metabolism employ microsomes, hepatocytes, and occasionally liver slices. It is a common practice to measure nonspecific binding to calculate unbound intrinsic clearance in microsomes (Obach, 1999; Austin et al., 2002). These studies typically employ equilibrium dialysis (over 5 hours) with a single low drug concentration (e.g., $1.0 \mu \mathrm{M})$ and varying $(0.3-10 \mathrm{mg} / \mathrm{ml})$ microsomal protein. Constant nonspecific binding and drug stability are assumed, but tissue uptake of drug results in a dilution effect during dialysis. When using hepatocytes, the incubation medium may contain no serum proteins, $10 \%$ fetal calf serum, or whole human serum (Riley et al., 2005). Drug binding in the cell cytosol is only sometimes measured (Naritomi et al., 2003). Again, single drug concentrations (1 $\mu \mathrm{M})$ are used, and constant, nonspecific binding is assumed. Equations using these data for prediction of in vivo clearances employ both the in vitro intrinsic clearances and FrUn values. The ability for making these predictions fare no better than yielding average differences of 4-fold (Riley et al., 2005; Wood et al., 2017). More rigorous attention to obtaining appropriate in vitro FrUn values has been called for (Wood et al., 2017).

\section{Summary}

Several conditions must be met for suitable application of diluted tissue homogenates along with the CFE (eq. 1) in measuring, predicting, and interpreting the tissue unbound fractions of drugs: 1) nonspecific and linear binding to macromolecules in plasma and tissues, along with lipid partitioning, dominate the tissue retention process; 2) drug distributes and binds relatively uniformly within each organ; 3) trapped blood drug contributions are either minor (e.g., brain) or are corrected for; 4) nonlinear processes, such as receptor binding, are noncontributory; 5) active influx or efflux transport do not contribute significantly; and 6) metabolic (elimination) processes and chemical instability are minimal. In vitro assessment of tissue binding or assessment of nonspecific binding using at least three dilutions or concentrations can help confirm linearity for extrapolation purposes. Some of these factors may contribute to the difficulty observed in using tissue homogenates to predict in vivo tissue partition coefficients and in assessing FrUn in microsomal and hepatocyte media. Although confirmation of tissue partition coefficients with in vivo studies is always advisable, the combined use of tissue preparations, in vivo measurements, and computational methods can provide unique insights into determinants of drug distribution (Ayyar et al., 2019b).

\section{Authorship Contributions}

Participated in research design: Jusko, Molins, Ayyar.

Conducted experiments: Molins, Ayyar.

Performed data analysis: Molins, Ayyar.

Wrote or contributed to the writing of the manuscript: Jusko, Ayyar

\section{References}

Agarwal V, Kommaddi RP, Valli K, Ryder D, Hyde TM, Kleinman JE, Strobel HW, and Ravindranath V (2008) Drug metabolism in human brain: high levels of cytochrome
P4503A43 in brain and metabolism of anti-anxiety drug alprazolam to its active metabolite. PLoS One 3:e2337.

Austin RP, Barton P, Cockroft SL, Wenlock MC, and Riley RJ (2002) The influence of nonspecific microsomal binding on apparent intrinsic clearance, and its prediction from physicochemical properties. Drug Metab Dispos 30:1497-1503.

Ayyar VS, DuBois DC, Nakamura T, Almon RR, and Jusko WJ (2019a) Modeling corticosteroid pharmacokinetics and pharmacodynamics, Part II: sex differences in methylprednisolone pharmacokinetics and corticosterone suppression. J Pharmacol Exp Ther 370:327-336.

Ayyar VS, Song D, DuBois DC, Almon RR, and Jusko WJ (2019b) Modeling corticosteroid pharmacokinetics and pharmacodynamics, Part I: determination and prediction of dexamethasone and methylprednisolone tissue binding in the rat. J Pharmacol Exp Ther 370:318-326.

Ballard BE (1974) Pharmacokinetics and temperature. J Pharm Sci 63:1345-1358.

Ballard PL, Baxter JD, Higgins SJ, Rousseau GG, and Tomkins GM (1974) General presence of glucocorticoid receptors in mammalian tissues. Endocrinology 94:998-1002.

Berezhkovskiy LM (2004) Volume of distribution at steady state for a linear pharmacokinetic system with peripheral elimination. J Pharm Sci 93:1628-1640.

Bernareggi A and Rowland M (1991) Physiologic modeling of cyclosporin kinetics in rat and man. J Pharmacokinet Biopharm 19:21-50.

Berry LM, Roberts J, Be X, Zhao Z, and Lin MH (2010) Prediction of V(ss) from in vitro tissuebinding studies. Drug Metab Dispos 38:115-121.

Boudinot FD, D'Ambrosio R, and Jusko WJ (1986) Receptor-mediated pharmacodynamics of prednisolone in the rat. J Pharmacokinet Biopharm 14:469-493.

Ferron GM and Jusko WJ (1998) Species differences in sirolimus stability in humans, rabbits, and rats. Drug Metab Dispos 26:83-84.

Fichtl B, Nieciecki A, and Walter K (1991) Tissue binding versus plasma binding of drugs: general principles and pharmacokinetic consequences. Adv Drug Res 20:117-166.

Fridén M, Bergström F, Wan H, Rehngren M, Ahlin G, Hammarlund-Udenaes M, and Bredberg U (2011) Measurement of unbound drug exposure in brain: modeling of $\mathrm{pH}$ partitioning explains diverging results between the brain slice and brain homogenate methods. Drug Metab Dispos 39: 353-362.

Fridén M, Gupta A, Antonsson M, Bredberg U, and Hammarlund-Udenaes M (2007) In vitro methods for estimating unbound drug concentrations in the brain interstitial and intracellular fluids. Drug Metab Dispos 35:1711-1719.

Fridén M, Ljungqvist H, Middleton B, Bredberg U, and Hammarlund-Udenaes M (2010) Improved measurement of drug exposure in the brain using drug-specific correction for residual blood. $J$ Cereb Blood Flow Metab 30:150-161.

Garg A and Balthasar JP (2009) Investigation of the influence of FcRn on the distribution of IgG to the brain. AAPS $J$ 11:553-557.

Goldstein A (1949) The interaction of drugs and plasma proteins. Pharmacol Rev 2:102-165.

Gomez-Zepeda D, Taghi M, Scherrmann JM, Decleves X, and Menet MC (2019) ABC transporters at the blood-brain interfaces, their study models, and drug delivery implications in glioma. Pharmaceutics 12:20.

Gustafson DL, Rastatter JC, Colombo T, and Long ME (2002) Doxorubicin pharmacokinetics: macromolecule binding, metabolism, and excretion in the context of a physiologic model. J Pharm Sci 91:1488-1501.

Haddad S, Poulin P, and Krishnan K (2000) Relative lipid content as the sole mechanistic determinant of the adipose tissue:blood partition coefficients of highly lipophilic organic chemicals. Chemosphere 40:839-843.

Harashima H, Sugiyama Y, Sawada Y, Iga T, and Hanano M (1984) Comparison between in-vivo and in-vitro tissue-to-plasma unbound concentration ratios $(\mathrm{Kp}, \mathrm{f})$ of quinidine in rats. $J$ Pharm Pharmacol 36:340-342.

Hazra A, Pyszczynski N, DuBois DC, Almon RR, and Jusko WJ (2007) Modeling receptor/genemediated effects of corticosteroids on hepatic tyrosine aminotransferase dynamics in rats: dual regulation by endogenous and exogenous corticosteroids. J Pharmacokinet Pharmacodyn 34:643-667. Hervé F, Urien S, Albengres E, Duché J-C, and Tillement J-P (1994) Drug binding in plasma. A summary of recent trends in the study of drug and hormone binding. Clin Pharmacokinet 26:44-58.

Jusko WJ and Gretch M (1976) Plasma and tissue protein binding of drugs in pharmacokinetics. Drug Metab Rev 5:43-140.

Kalvass JC and Maurer TS (2002) Influence of nonspecific brain and plasma binding on CNS exposure: implications for rational drug discovery. Biopharm Drug Dispos 23:327-338.

Kalvass JC, Maurer TS, and Pollack GM (2007) Use of plasma and brain unbound fractions to assess the extent of brain distribution of 34 drugs: comparison of unbound concentration ratios to in vivo p-glycoprotein efflux ratios. Drug Metab Dispos 35:660-666.

Kalvass JC, Phipps C, Jenkins GJ, Stuart P, Zhang X, Heinle L, Nijsen MJMA, and Fischer V (2018) Mathematical and experimental validation of flux dialysis method: an improved approach to measure unbound fraction for compounds with high protein binding and other challenging properties. Drug Metab Dispos 46:458-469.

Khor SP and Mayersohn M (1991) Potential error in the measurement of tissue to blood distribution coefficients in physiological pharmacokinetic modeling. Residual tissue blood. I. Theoretical considerations. Drug Metab Dispos 19:478-485.

Landersdorfer CB, He YL, and Jusko WJ (2012) Mechanism-based population pharmacokinetic modelling in diabetes: vildagliptin as a tight binding inhibitor and substrate of dipeptidyl peptidase IV. Br J Clin Pharmacol 73:391-401.

Lin JH (2006) Tissue distribution and pharmacodynamics: a complicated relationship. Curr Drug Metab 7:39-65.

Lin JH, Sugiyama Y, Awazu S, and Hanano M (1982) In vitro and in vivo evaluation of the tissueto-blood partition coefficient for physiological pharmacokinetic models. J Pharmacokinet Biopharm 10:637-647.

Longhi R, Corbioli S, Fontana S, Vinco F, Braggio S, Helmdach L, Schiller J, and Boriss H (2011) Brain tissue binding of drugs: evaluation and validation of solid supported porcine brain membrane vesicles (TRANSIL) as a novel high-throughput method. Drug Metab Dispos 39: 312-321.

Loryan I, Melander E, Svensson M, Payan M, König F, Jansson B, and Hammarlund-Udenaes M (2016) In-depth neuropharmacokinetic analysis of antipsychotics based on a novel approach to estimate unbound target-site concentration in CNS regions: link to spatial receptor occupancy. Mol Psychiatry 21:1527-1536.

Mager DE and Jusko WJ (2001) General pharmacokinetic model for drugs exhibiting targetmediated drug disposition. J Pharmacokinet Pharmacodyn 28:507-532.

Mendel CM (1989) The free hormone hypothesis: a physiologically based mathematical model. Endocr Rev 10:232-274. 
Naritomi Y, Terashita S, Kagayama A, and Sugiyama Y (2003) Utility of hepatocytes in predicting drug metabolism: comparison of hepatic intrinsic clearance in rats and humans in vivo and in vitro. Drug Metab Dispos 31:580-588.

Obach RS (1999) Prediction of human clearance of twenty-nine drugs from hepatic microsomal intrinsic clearance data: an examination of in vitro half-life approach and nonspecific binding to microsomes. Drug Metab Dispos 27:1350-1359.

O'Brien JS and Sampson EL (1965) Lipid composition of the normal human brain: gray matter, white matter, and myelin. J Lipid Res 6:537-544.

Poulin P (2015) A paradigm shift in pharmacokinetic-pharmacodynamic (PKPD) modeling: rule of thumb for estimating free drug level in tissue compared with plasma to guide drug design. J Pharm Sci 104:2359-2368.

Poulin P and Theil FP (2000) A priori prediction of tissue:plasma partition coefficients of drugs to facilitate the use of physiologically-based pharmacokinetic models in drug discovery. J Pharm Sci 89:16-35.

Poulin P and Theil FP (2002) Prediction of pharmacokinetics prior to in vivo studies. II. Generic physiologically based pharmacokinetic models of drug disposition. J Pharm Sci 91:1358-1370.

Riley RJ, McGinnity DF, and Austin RP (2005) A unified model for predicting human hepatic, metabolic clearance from in vitro intrinsic clearance data in hepatocytes and microsomes. Drug Metab Dispos 33:1304-1311.

Rodgers T, Jones HM, and Rowland M (2012) Tissue lipids and drug distribution: dog versus rat. J Pharm Sci 101:4615-4626.

Rodgers T and Rowland M (2006) Physiologically based pharmacokinetic modelling 2: predicting the tissue distribution of acids, very weak bases, neutrals and zwitterions. J Pharm Sci 95:1238-1257.
Stieger B and Gao B (2015) Drug transporters in the central nervous system. Clin Pharmacokinet 54:225-242.

Taves MD, Schmidt KL, Ruhr IM, Kapusta K, Prior NH, and Soma KK (2010) Steroid concentrations in plasma, whole blood and brain: effects of saline perfusion to remove blood contamination from brain. PLoS One 5:e15727.

Trapp S, Rosania GR, Horobin RW, and Kornhuber J (2008) Quantitative modeling of selective lysosomal targeting for drug design. Eur Biophys $J$ 37:1317-1328.

Wan H, Rehngren M, Giordanetto F, Bergström F, and Tunek A (2007) High-throughput screening of drug-brain tissue binding and in silico prediction for assessment of central nervous system drug delivery. J Med Chem 50:4606-4615.

Wood FL, Houston JB, and Hallifax D (2017) Clearance prediction methodology needs fundamental improvement: trends common to rat and human hepatocytes/microsomes and implications for experimental methodology. Drug Metab Dispos 45:1178-1188.

Yokogawa K, Ishizaki J, Ohkuma S, and Miyamoto K (2002) Influence of lipophilicity and lysosomal accumulation on tissue distribution kinetics of basic drugs: a physiologically based pharmacokinetic model. Methods Find Exp Clin Pharmacol 24:81-93.

Address correspondence to: Dr. William J. Jusko, Department of Pharmaceutical Sciences, School of Pharmacy and Pharmaceutical Sciences, University at Buffalo, Buffalo, NY 14214. E-mail: wjjusko@buffalo.edu 